\title{
Strains of Lactobacillus plantarum in Grape Must are also Present in the Intestinal Tract of Vinegar Flies
}

\author{
W.H. Groenewald, C.A. van Reenen and L.M.T. Dicks* \\ Department of Microbiology, Stellenbosch University, Private Bag X1, 7602 Matieland (Stellenbosch), South Africa
}

Submitted for publication: November 2005

Accepted for publication: April 2006

Key words: Lactobacillus plantarum, vinegar flies

\begin{abstract}
Twenty-one lactic acid bacteria isolated from the intestinal tract of Drosophila simulans Stuvervant and nine from Merlot noir grapes were identified as $L$. plantarum by PCR with species-specific primers and 16S rDNA sequencing. The 30 isolates grouped into four clusters based on RAPD-PCR banding patterns, suggesting that they belong to at least four genotypic groups. Thirteen isolates from grape must and five from the flies yielded identical RAPDPCR banding patterns and grouped into one cluster, suggesting that they are descendants from the same strain. Concluded from these results, $L$. plantarum (or at least descendants from a specific strain) has the ability to use vinegar flies as a host and vector to infect grape must. Further research is needed to determine the role of this specific strain in wine fermentations.
\end{abstract}

\section{INTRODUCTION}

Lactobacillus plantarum is one of the most widely distributed lactic acid bacteria, probably due to its ability to adapt to various niches. The species is commonly isolated from grape must (Davis et al., 1985; Du Plessis et al., 2004) and is present in fairly high cell numbers during the first few days of fermentation. Although the cell numbers of L. plantarum usually decreases during secondary fermentation, some strains withstand the high $\mathrm{SO}_{2}$ and ethanol concentrations and may cause spoilage of bottled wine (Davis et al., 1985; Du Toit \& Pretorius, 2000). A few strains of L. plantarum with strong malolactic fermentation have been used as starter cultures (Davis et al., 1985).

Drosophila is a common agricultural pest. The flies lay their eggs on fruit, which nourishes the developing larvae (Demerec, 1950). A number of yeasts have been isolated from the intestinal tract of Drosophila (Mortimer and Polsinelli, 1999; Ganter, 2006). Wolbachia, a rickettsia-like organism (Rousset et al., 1992) and Vibrio cholerae (Park et al., 2005) have also been isolated from Drosophila. However, little is known about the presence of lactic acid bacteria in the gut of vinegar flies. Kvasnikov et al. (1971) reported the presence of $L$. plantarum and enterococci in Drosophila and suggested that they may contaminate fermentation processes. The latter strains were identified based on physiological and biochemical characteristics (Kvasnikov et al., 1971), including sugar fermentation profiles that are often not reliable (Van Reenen \& Dicks, 1996; Kullen et al., 1998). In a recent study (Groenewald, 2005), L. plantarum (70\%), Lactobacillus paracasei (3.3\%), Lactobacillus sanfranciscensis (6.7\%), Leuconostoc mesenteroides subsp. mesenteroides (3.3\%), Lactococcus lactis subsp. lactis (3.3\%), Enterococcus faecalis $(6.7 \%)$ and Pediococcus pentosaceus $(6.7 \%)$ have been isolated from vinegar flies.
The role of lactic acid bacteria in the insect gut is not known. They may be involved in the detoxification of plant allelochemicals such as flavonoids, tannins, and alkaloids (Dillon \& Dillon, 2004), or prevent the colonisation of non-indigenous pathogenic micro-organisms by competitive exclusion (Berg, 1996).

This study was conducted to confirm the presence of L. plantarum in vinegar flies and to determine if the species can use the insect as a vector.

\section{MATERIALS AND METHODS}

\section{Collection of samples}

Five kilograms of Merlot noir grapes collected from a vineyard in Stellenbosch were crushed in sterile plastic bags and the must removed aseptically. Vinegar flies were captured from the same vineyard using specially designed traps. The flies were sterilised with $2.5 \%$ ( $\mathrm{vol} / \mathrm{vol}$ ) sodium hypochlorite and rinsed several times with sterile distilled water. Water from the last washing was inoculated onto MRS agar (Biolab, Biolab Diagnostics, Midrand, SA) to evaluate the efficiency of the washing process. The plates were incubated at $30^{\circ} \mathrm{C}$ and examined for microbial growth after $48 \mathrm{~h}$.

Approximately 100 flies were placed in a sterile $50 \mathrm{~mL}$ centrifuge tube with $2 \mathrm{~mL}$ sterile peptone water and glass beads (approximately $2 \mathrm{~mm}$ in diameter). The flies were homogenised for $3 \mathrm{~min}$ at $25^{\circ} \mathrm{C}$ on a vortex. The homogenate was serially diluted in sterile distilled water and plated out, in triplicate, onto MRS agar (Biolab), supplemented with $20 \%$ (vol/vol) apple juice and adjusted to $\mathrm{pH} 5.5$ with $1 \mathrm{~N} \mathrm{NaOH}$. The medium was supplemented with $100 \mu \mathrm{g} / \mathrm{mL}$ Delvocid (GistBrocades, Delft, Netherlands) to inhibit the growth of yeast and fungi. One set of plates was incubated in an anaerobic flask (Oxoid, Basingstoke, Hampshire, England) in the presence of an Anaerocult gas generating kit (Oxoid). The

*Corresponding author: E-mail address: lmtd@sun.ac.za

Acknowledgements: This research was funded by the National Research Foundation (NRF), South Africa. 
remaining two sets of plates were incubated aerobically at $30^{\circ} \mathrm{C}$. All plates were examined for growth after 24 and $48 \mathrm{~h}$.

\section{Preliminary identification of the isolates}

Colonies were randomly selected from plates between 50 and 300 colonies and re-streaked on corresponding media to obtain pure cultures. All cultures were stored at $-80^{\circ} \mathrm{C}$ in MRS broth supplemented with sterile glycerol (30\%, vol/vol, final concentration). Gram reaction and catalase activity were determined according to the methods described by Harrigan and McCance (1976).
Production of $\mathrm{CO}_{2}$ from glucose and gluconate was monitored according to the method described by Dicks and Van Vuuren (1987). Facultative heterofermentative, Gram-positive and catalase negative rods were selected and the configuration of lactic acid produced determined by using an enzymatic kit (Roche Diagnostics GmbH, Mannheim, Germany).

\section{Carbohydrate fermentations}

Carbohydrate fermentation reactions were recorded by using the API 50 CHL system (BioMerieux, Marcy L'Etoile, France). All

\section{TABLE 1}

Differential carbohydrate fermentation reactions of Lactobacillus plantarum isolates collected from vinegar flies and grape must. Isolates from grape must are printed in bold.

\begin{tabular}{|c|c|c|c|c|c|c|c|c|c|c|c|c|c|c|c|}
\hline Isolate & $\frac{\bar{d}}{\stackrel{0}{0}}$ & 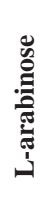 & $\frac{\ddot{\partial}}{\grave{\partial}}$ & 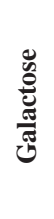 & 导 & 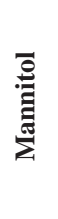 & 司 & 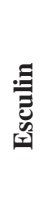 & 总 & 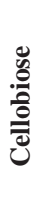 & 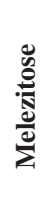 & 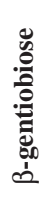 & 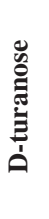 & 营 & 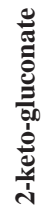 \\
\hline L. plantarum ATCC $14917^{\mathrm{Ta}}$ & - & + & - & + & - & + & + & + & + & + & + & + & - & + & - \\
\hline 32 & $\mathrm{~d}$ & + & - & + & $\mathrm{d}$ & + & + & + & + & + & + & + & - & + & - \\
\hline 26 & $\mathrm{~d}$ & + & - & + & $\mathrm{d}$ & + & + & + & + & + & + & + & $\mathrm{d}$ & + & - \\
\hline $3 \mathrm{~A} 1$ & - & + & - & + & $\mathrm{d}$ & + & + & + & + & + & + & + & + & + & - \\
\hline $\mathrm{PP}$ & - & + & - & + & $\mathrm{d}$ & + & + & + & + & + & + & + & $\mathrm{d}$ & $\mathrm{d}$ & $\mathrm{D}$ \\
\hline $\mathrm{R} 2$ & - & + & - & + & $\mathrm{d}$ & + & + & + & + & + & + & + & $\mathrm{d}$ & + & - \\
\hline 37 & - & - & - & $\mathrm{d}$ & - & + & - & $\mathrm{d}$ & $\mathrm{d}$ & $\mathrm{d}$ & $\mathrm{d}$ & + & - & $\mathrm{d}$ & - \\
\hline 28 & - & - & - & $\mathrm{d}$ & $\mathrm{d}$ & + & + & + & + & + & + & + & - & + & - \\
\hline $\mathrm{E}$ & $\mathrm{d}$ & + & $\mathrm{d}$ & + & $\mathrm{d}$ & + & + & + & + & + & + & $\mathrm{d}$ & - & $\mathrm{d}$ & - \\
\hline $\mathrm{AA}$ & - & - & - & $\mathrm{d}$ & - & + & - & + & $\mathrm{d}$ & $\mathrm{d}$ & $\mathrm{d}$ & + & - & $\mathrm{d}$ & - \\
\hline $\mathrm{U} 2$ & $\mathrm{~d}$ & + & - & + & $\mathrm{d}$ & + & + & + & + & + & + & + & + & + & - \\
\hline 24 & - & - & - & $\mathrm{d}$ & - & + & - & + & + & $\mathrm{d}$ & $\mathrm{d}$ & + & - & $\mathrm{d}$ & - \\
\hline A7 & $\mathrm{d}$ & + & - & + & $\mathrm{d}$ & + & + & + & + & + & + & + & + & + & - \\
\hline FA5 & $\mathrm{d}$ & + & - & + & $\mathrm{d}$ & + & + & + & + & + & + & + & $\mathrm{d}$ & $\mathrm{d}$ & $\mathrm{d}$ \\
\hline A1 & - & + & - & + & $\mathrm{d}$ & + & + & + & + & + & + & + & - & + & - \\
\hline FA13 & - & + & - & + & - & + & + & + & + & + & + & + & - & $\mathrm{d}$ & - \\
\hline 27 & $\mathrm{~d}$ & + & - & + & $\mathrm{d}$ & + & + & + & + & + & + & + & $\mathrm{d}$ & + & - \\
\hline MII & - & - & - & $\mathrm{d}$ & $\mathrm{d}$ & + & + & + & + & + & + & + & - & + & - \\
\hline M1 & - & + & - & + & $\mathrm{d}$ & + & + & + & + & + & + & + & - & + & - \\
\hline F10 & $\mathrm{d}$ & + & - & + & $\mathrm{d}$ & + & + & + & + & + & + & + & $\mathrm{d}$ & $\mathrm{d}$ & $\mathrm{D}$ \\
\hline 21 & - & + & - & + & $\mathrm{d}$ & + & + & + & + & + & + & + & $\mathrm{d}$ & + & - \\
\hline 34 & $\mathrm{~d}$ & + & $\mathrm{d}$ & + & $\mathrm{d}$ & + & + & + & + & + & + & $\mathrm{d}$ & - & $\mathrm{d}$ & - \\
\hline C8 & - & + & - & + & $\mathrm{d}$ & + & + & + & + & + & + & + & - & $\mathrm{d}$ & - \\
\hline C1 & - & + & - & + & $\mathrm{d}$ & + & + & + & + & + & + & + & $\mathrm{d}$ & + & $\mathrm{D}$ \\
\hline C32 & - & + & - & + & $\mathrm{d}$ & + & + & + & + & + & + & + & - & $\mathrm{d}$ & - \\
\hline CA4 & $\mathrm{d}$ & + & - & + & $\mathrm{d}$ & + & + & + & + & + & + & + & - & $\mathrm{d}$ & D \\
\hline C23 & - & + & - & + & $\mathrm{d}$ & + & + & + & + & + & + & + & $\mathrm{d}$ & + & - \\
\hline $3 \mathrm{C} 3$ & $\mathrm{~d}$ & + & $\mathrm{d}$ & + & $\mathrm{d}$ & + & + & + & + & + & + & + & - & + & - \\
\hline C3 & - & - & - & + & - & + & - & + & $\mathrm{d}$ & $\mathrm{d}$ & $\mathrm{d}$ & + & - & $\mathrm{d}$ & - \\
\hline $\mathrm{C} 12$ & $\mathrm{~d}$ & + & - & + & $\mathrm{d}$ & - & + & + & + & + & + & + & + & + & - \\
\hline C13 & - & - & - & $\mathrm{d}$ & - & + & - & + & + & + & $\mathrm{d}$ & + & - & $\mathrm{d}$ & - \\
\hline
\end{tabular}

+ , positive reaction; -, negative reaction; d, variable reaction. All strains fermented: $N$-acetyl-glucosamine, amygdalin, arbutin, D-fructose, D-glucose, lactose, maltose, Dmannose, melibiose, raffinose, ribose, saccharose and trehalose. None of the strains fermented adonitol, D-arabinose, D-arabitol, L-arabitol, dulcitol, erythritol, D-fucose, L-fucose, glycogen, inositol, inulin, 5-keto-gluconate, D-lyxose, $\alpha$-methyl-D-glucoside , $\alpha$-methyl-D-mannoside, $\beta$-methyl-xyloside, L-sorbose, starch, D-tagatose, xylitol and L-xylose.

a Data from Kandler and Weiss (1986). 
API strips were incubated at $30^{\circ} \mathrm{C}$ and readings were taken after 24 and $48 \mathrm{~h}$, respectively.

\section{PCR with species-specific primers}

Isolates with carbohydrate fermentation reactions corresponding to that of the type strain of L. plantarum (ATCC 14917) were selected and their genomic DNA isolated according to the method described by Dellaglio et al. (1973). The DNA was amplified with primers planF (CCG TTT ATG CGG AAC ACC TA) and REV (TCG GGA TTA CCA AAC ATC AC), and Taq Takara polymerase (Otsu, Shiga, Japan), according to the method used by Torriani et al. (2001). L. plantarum ATCC $14917^{\mathrm{T}}$ was used as reference strain.

\section{Sequencing of $16 \mathrm{~S}$ rDNA}

Isolates with DNA fragments identical in size to that of L. plantarum ATCC $14917^{\mathrm{T}}$ were selected and their genomic DNA annealed to primers $8 \mathrm{f}$ (5'-CAG GGA TCC AGA CTT TGA TYM TGG CTC AG-3') and 1512r (5'-GTG AAG CTT ACG GYT AGC TTG TTA CGA CTT-3') to amplify a region in the 16S rDNA molecule. The methods of Felske et al. (1997) and Garbers et al. (2004) were used, but with the Taq Takara polymerase.

The amplified fragments were purified using the High Pure PCR product Purification Kit (Roche), according to the manufacturer's instructions. The fragments were sequenced and compared with sequences in Genbank by using the BLAST programme.

\section{RAPD-PCR analysis}

Genomic DNA from the isolates was amplified with eight decamer primers, (OPL-01, OPL-02, OPL-03, OPL-04, OPL-05, OPL-08, OPL-12, OPL-20), as described by Van Reenen and Dicks (1996). The primers were of arbitrary sequence from the OPL set (Operon Technologies Inc., Alameda, CA, USA). Taq Supertherm polymerase (Hoffmann-La Roche, Nutley, NJ) was used.

\section{RESULTS AND DISCUSSION}

No viable micro-organisms were isolated from the water washings, indicating that all surface-bound cells had been washed off. Plates incubated anaerobically displayed less growth than those incubated aerobically.

From a total of 158 isolates, 30 were Gram-positive, catalase negative and produced $\mathrm{CO}_{2}$ from D-gluconate, but not from Dglucose. Based on these characteristics, the isolates were classified as members of Group II (facultatively heterofermentative) Lactobacillus spp. All strains produced DL-lactate from D-glucose. Carbohydrate fermentation reactions corresponded to that recorded for the type strain of L. plantarum (ATCC 14917T, Table 1). Variations in the fermentation of L-arabinose, cellobiose, esculin, galactose, $\beta$-gentiobiose, gluconate, glycerol, 2 -keto-gluconate, mannitol, melezitose, rhamnose, salicin, sorbitol, D-turanose and D-xylose were recorded (see Table 1). Similar results have been reported for other strains of L. plantarum (Zanoni, 1987; Van Reenen \& Dicks, 1996). None of the strains fermented $\alpha$-methyl-D-mannoside, which is characteristic for the type strain of L. plantarum, ATCC $14917^{\mathrm{T}}$ (Zanoni, 1987; Van Reenen \& Dicks, 1996). Based on carbohydrate fermentation reactions, the isolates were preliminary classified as strains of L. plantarum. Sugar fermentation reactions did not show correlation with
RAPD groupings. Strains of L. plantarum have been isolated from Drosophila (Kvasnikov et al., 1971). However, the genotypic relatedness of these strains has not been studied. Comparisons with the latter strains are not possible, since they have not been deposited into a culture collection.

Amplification of genomic DNA with species-specific primers yielded a 318-bp fragment, which is identical in size to that reported for L. plantarum ATCC $14917^{\mathrm{T}}$ (Torriani et al., 2001). Furthermore, amplification of the genomic DNA of the isolates with primers $8 \mathrm{f}$ and $1512 \mathrm{r}$ yielded $16 \mathrm{~S}$ rDNA amplicons that were $97.1 \%$ (832 of 857 bp sequenced) to $99.0 \%$ (849 of 858 bp sequenced) homologous to the $16 \mathrm{~S}$ rDNA of $L$. plantarum WCFS1 (NCBI nucleotide sequence database, accession number AL935258.1), confirming their classification as L. plantarum.

Identical RAPD-PCR profiles were recorded with primers OPL-01, OPL-02 and OPL-12. Primer OPL-20 produced an incomplete profile, primer OPL-03 two different profiles, and primer OPL-08 three different profiles (results not shown). Similar results were recorded in a previous study with 27 different strains of L. plantarum (Van Reenen \& Dicks, 1996). Amplification with primers OPL-04 and OPL-05 grouped the 30 isolates into four genotypically well-separated clusters (see Fig. 1). Identical DNA profiles were recorded with duplicate RAPDPCR runs. Isolates in clusters I and II were from vinegar flies and isolates in cluster IV from grape must. Cluster III contained five isolates $(\mathrm{C} 4, \mathrm{C} 1, \mathrm{C} 32, \mathrm{C} 23$ \& 3C3) from vinegar flies and 13 from grape must. The identical DNA banding patterns obtained for all the isolates in cluster III suggests that they are descendants from the same strain, but genotypically different from the type strain of L. plantarum (ATCC $14917^{\mathrm{T}}$ ) which did not group in any of the four RAPD-PCR clusters (see Fig. 1). This also suggests that at least one strain of L. plantarum developed the ability to colonise the intestinal tract of $D$. simulans Stuvervant and use the insect as a vector. Insects are known to serve as vectors for bacteria (Lilley et al., 1997). As far as we could determine, this is the first evidence of vinegar flies acting as a vector for L. plantarum. Further research is needed to determine the role of this specific strain (isolates from cluster III, Fig. 1) in wine fermentations.

\section{TABLE 2}

Percentage similarity of isolates to species in the NCBI nucleotide sequence database, based on partial 16S rDNA sequence analysis. Isolates from grape must are printed in bold.

\begin{tabular}{llc}
\hline Isolate & Phylogenetic affiliation and accession number & $\begin{array}{c}\% \\
\text { similarity }\end{array}$ \\
\hline AA & Lactobacillus plantarum (AL935258.1) & $99.0 \%$ \\
A1 & Lactobacillus plantarum (AL935258.1) & $99.0 \%$ \\
21 & Lactobacillus plantarum (AL935258.1) & $98.8 \%$ \\
CA4 & Lactobacillus plantarum (AL935258.1) & $98.8 \%$ \\
FA13 & Lactobacillus plantarum (AL935258.1) & $98.8 \%$ \\
C1 & Lactobacillus plantarum (AL935258.1) & $98.7 \%$ \\
26 & Lactobacillus plantarum (AL935258.1) & $98.6 \%$ \\
E & Lactobacillus plantarum (AL935258.1) & $98.4 \%$ \\
C8 & Lactobacillus plantarum (AL935258.1) & $98.2 \%$ \\
R2 & Lactobacillus plantarum (AL935258.1) & $98.2 \%$ \\
MII & Lactobacillus plantarum (AL935258.1) & $97.1 \%$ \\
\hline
\end{tabular}




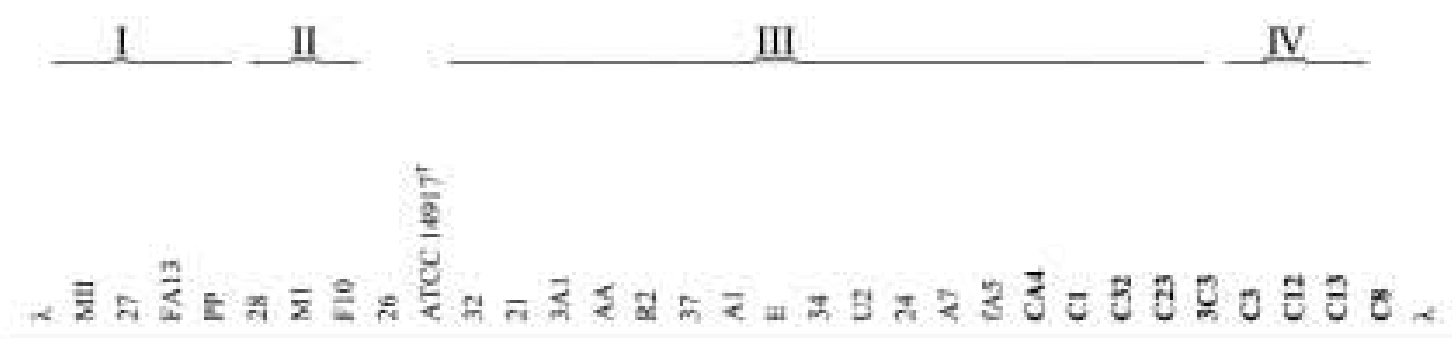

A
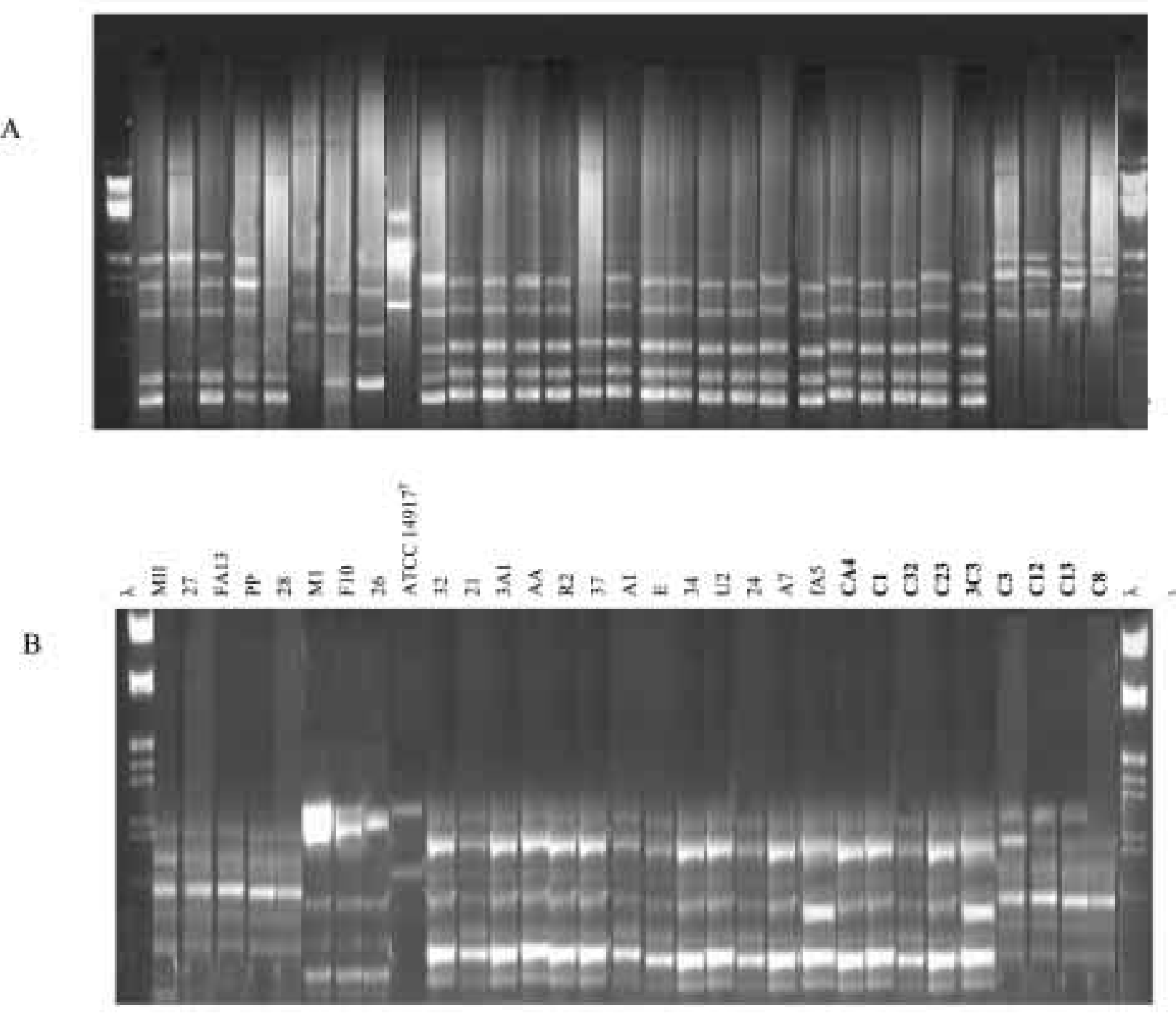

FIGURE 1

DNA fragments obtained after RAPD-PCR of the genomic DNA of isolates identified as Lactobacillus plantarum. A: Primer OPL-4(GACTGCACAC), B: Primer OPL5 (ACGCAGGCAC).

\section{LITERATURE CITED}

Berg, R.D., 1996. The indigenous gastrointestinal microflora. Trends Microbiol. 4, 430-435.

Davis, C.R., Wibowo, D., Eschenbruch, R., Lee, T.H. \& Fleet, G.H. 1985. Practical implications of malolactic fermentation. A review. Am. J. Enol. Vitic. 36, 290-301.

Dellaglio, F., Bottazzi, V. \& Trovatelli, L.D., 1973. Deoxyribonucleic acid homology and base composition in some thermophilic lactobacilli. J. Gen. Microbiol. 74, 289-297.

Demerec, M. , 1950 (1st ed). Biology of Drosophila. John Wiley \& Sons, New York.
Dicks, L.M.T. \& Van Vuuren, H.J.J., 1987. A modification of the hot tube method for the detection of carbon dioxide produced by heterofermentative Lactobacillus strains. J. Microbiol. Meth. 6, 273-275.

Dillon, R.J. \& Dillon, V.M., 2004. Gut bacteria of insects. Nonpathogenic interactions. Ann. Rev. Entomol. 49, 71-92.

Du Plessis, H.W., Dicks, L.M.T., Pretorius, I.S., Lambrechts, M.G. \& du Toit, M., 2004. Identification of lactic acid bacteria isolated from South African brandy base wines. Int. J. Food Microbiol. 91, 19-29.

Du Toit, M.G. \& Pretorius, I.S., 2000. Microbial spoilage and preservation of wine: Using weapons from nature's own arsenal - A review. S. Afr. J. Enol. Vitic. 21, 74-96. 
Felske, A., Rheims, H., Wolterink, A., Stackebrant, E. \& Akkermans, A.D.L., 1997. Ribosome analysis reveals prominent activity of an uncultured member of the class Acinetobacteria in grasslands soil. Microbiol. 143, 2983-2989.

Ganter, P.F., 2006. Yeast and invertebrate associations. In: Rosa, C. \& Péter, G. (eds). Biodiversity and ecophysiology of yeasts. Springer. pp. 303-370.

Garbers, I-M., Britz, T.J. \& Witthuhn, R.C., 2004. PCR-based denaturing gradient gel electrophoretic typification and identification of microbial consortiums present in Kefir grains. World J. Microbiol. Biotechnol. 20, 687-693.

Groenewald, W.H., 2005. A taxonomic study on lactic acid bacteria isolated from fruit flies. MSc. thesis, University of Stellenbosch.

Harrigan, W.F. \& McCance, M.E., 1976 (1st ed). Laboratory Methods in Food and Dairy Microbiology. Academic Press, New York.

Kandler, O. \& Weiss, N., 1986. Regular, nonsporing Gram-positive rods. In: Sneath, P.H.A., Mair, N., Sharpe, M.E., \& Holt, J.G. (eds). Bergey's manual of systematic bacteriology, vol II. Williams \& Wilkins, Baltimore. pp. 1208-1234.

Kullen, M.J., Khil, J., Busta, F.F., Gallaher, D.D. \& Brady, L.J., 1998. Carbohydrate source and bifidobacteria influence the growth of Clostridium perfringens in vivo and in vitro. Nutr. Res. 18, 1889-1897.

Kvasnikov, E.I., Kovalenko, N.K. \& Nesterenko, O.A., 1971. Lactic acid bacteria in insects. Mikrobiologia 40, 144-151.
Lilley, A.K., Hails, R.S., Cory, J.S. \& Bailey, M.J., 1997. The dispersal and establishment of pseudomonad populations in the phyllosphere of sugar beet by phytophagous caterpillars. FEMS Microbiol. Ecol. 24, 151-57.

Mortimer, R. \& Polsinelli, M., 1999. On the origins of wine yeast. Res. Microbiol. $150,199-204$

Park, S.Y., Heo, Y.J, Kim, K.S. \& Cho, Y.H., 2005. Drosophila melanogaster is susceptible to Vibrio cholerae infection. Mol Cells 20, 409-415.

Rousset, F., Vautrin, D. \& Solignac, M., 1992. Molecular identification of Wolbachia, the agent of cytoplasmic incompatibility in Drosophila simulans, and variability in relation with host mitochondrial types. Proc. Biol. Sci. 247, 163-168.

Torriani, S., Felis, G.E. \& Dellaglio, F., 2001. Differentiation of Lactobacillus plantarum, Lactobacillus pentosus, and Lactobacillusd paraplantarum by recA gene sequence analysis and multiplex PCR assay with $r e c A$ gene-derived primers. Appl. Environ. Microbiol. 67, 3450-3454.

Van Reenen, C.A. \& Dicks, L.M.T., 1996. Evaluation of numerical analysis of random amplified polymorphic DNA (RAPD)-PCR as a method to differentiate Lactobacillus plantarum and Lactobacillus pentosus. Curr. Microbiol. 32, 183-187.

Zanoni, P., Farrow, J.A.E., Phillips, B.A. \& Collins, M.D., 1987. Lactobacillus pentosus (Fred, Peterson and Anderson) sp. nov., nom. rev. Int. J. Syst. Bacteriol. 37, 339-341. 